WEIF-8

\title{
Full-Wave Characterization of an Edge-Coupled Coplanar-Waveguide Structure with Backed Conductor
}

\author{
Chun-Lin Liao and Chun Hsiung Chen \\ Department of Electrical Engineering and Graduate Institute \\ of Communication Engineering, National Taiwan University, Taipei 106, Taiwan
}

\begin{abstract}
A novel edge-coupled coplanarwaveguide (CPW) structure with finite-extent backed conductor is proposed. Based on the full-wave analysis, the effective dielectric constants and characteristic impedances of odd and even CPW modes are characterized, together with the associated coupling coefficients. The electric field distributions of these two modes are also presented.
\end{abstract}

\section{INTRODUCTION}

The coupling effect between two coplanar waveguides (CPW) on the same side of the substrate is relatively small due to the presence of the central ground plane which prevents the two lines to close to each other. To increase the coupling effect, an edgecoupled coplanar-waveguide structure without the central ground plane was proposed [1]. Based on the quasistatic design parameters, a $10 d B$ (CPW directional coupler was implemented [1], and the associated effective dielectric constants based on the full-wave analysis were presented [2]. In order to improve the coupling characteristics between

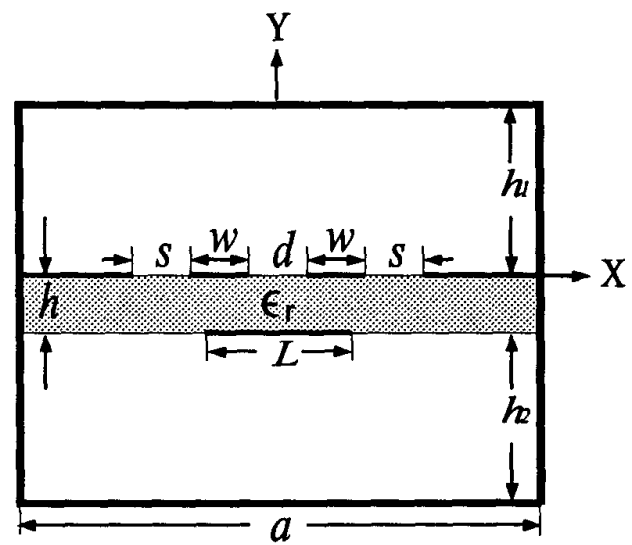

Fig. 1. Cross section of conductor-backed edge-coupled CPW structure $(w=s=$ $h=200 \mu m, \epsilon_{r}=16, h_{1}=h_{2}=10 \mathrm{~mm}$, $a=20 \mathrm{~mm}$ ).

the two CPW's, a novel edge-coupled CPW structure with backed conductor is proposed in this paper and is then analyzed by the full-wave method.

\section{FORMULATION}

Consider a conductor-backed edgecoupled CPW structure as shown in Fig. 1 in which a backed conductor of width $L$ is incorporated into the other side of the sub- 
strate. 'I'his structure has identical strip width $w$ and slot width $s$, and the dielectric constant and thickness of the substrate are $\epsilon_{r}$ and $h$, respectively. The coupling mechanism is controlled by the spacing $d$ between the strips and also by the width $L$ of backed conductor. To simplify the analysis, the proposed CPW coupled structure is enclosed by a conducting box.

Although three fundamental modes are supported by the four-conductor system (Fig. 1), we are mainly concerned with the odd CPW mode and even CPW mode whose characteristics may be analyzed by introducing the electric wall and magnetic wall on the plane $x=0$. These two CPW modes are responsible for the coupling mechanism associated with the coupled structure.

By using the spectral-domain method, the effeclive dielectric constants $t_{e f f}$ and characteristic impedances $Z_{0}$ for both odd and even CPW modes are calculated and presented. The structure without a backed conductor $(L=0)$ reduces to the conventional edge-coupled CPW structure as discussed by [1].

\section{NUMERICAL RESULTS}

Fig. 2 shows the effective dielectric constants and characteristic impedances for odd and even CPW modes as well as the associated coupling coefficient $C=20 \times$ $\log \left[\left(Z_{o}^{\text {cven }}-Z_{o}^{\text {odd }}\right) /\left(Z_{o}^{\text {cven }}+Z_{o}^{\text {odd }}\right)\right]$ for the conventional edge-coupled $\mathrm{CPW}$ structure $(L=0)$. The coupling coefficient increases when the spacing $d$ between strips decreases. The coupling is not strong, and the spacing must reduce to $50 \mu \mathrm{m}$ so as to achieve a $-8 d B$ coupling coefficient. Such a. short

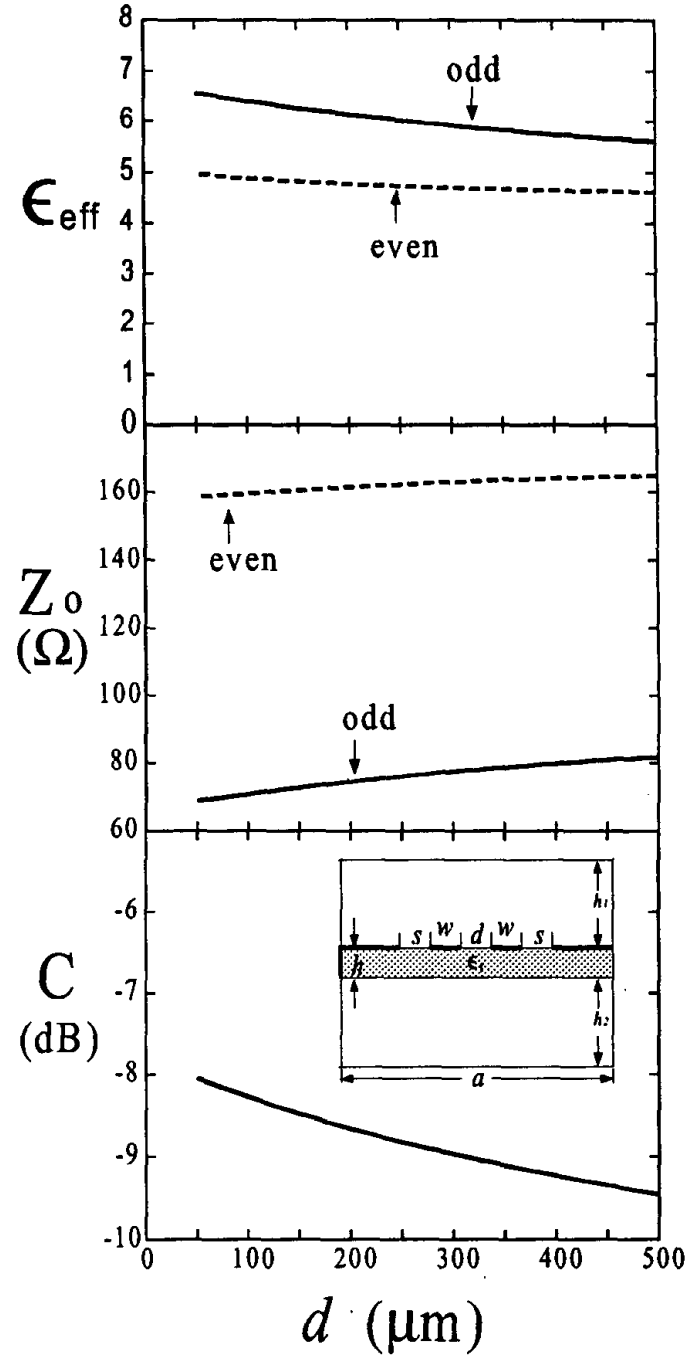

Fig. 2. Eflect of line spacing $d$ on cffcctive diclectric constants, charactcristic impedances, and coupling coefficient of the cdge-coupled CPW structure without a. backed conductor $(f=3 G H z)$.

spacing may suffer a larger conductor loss [3]. To increase the coupling effect of the conventional edge-coupled CPW structure 


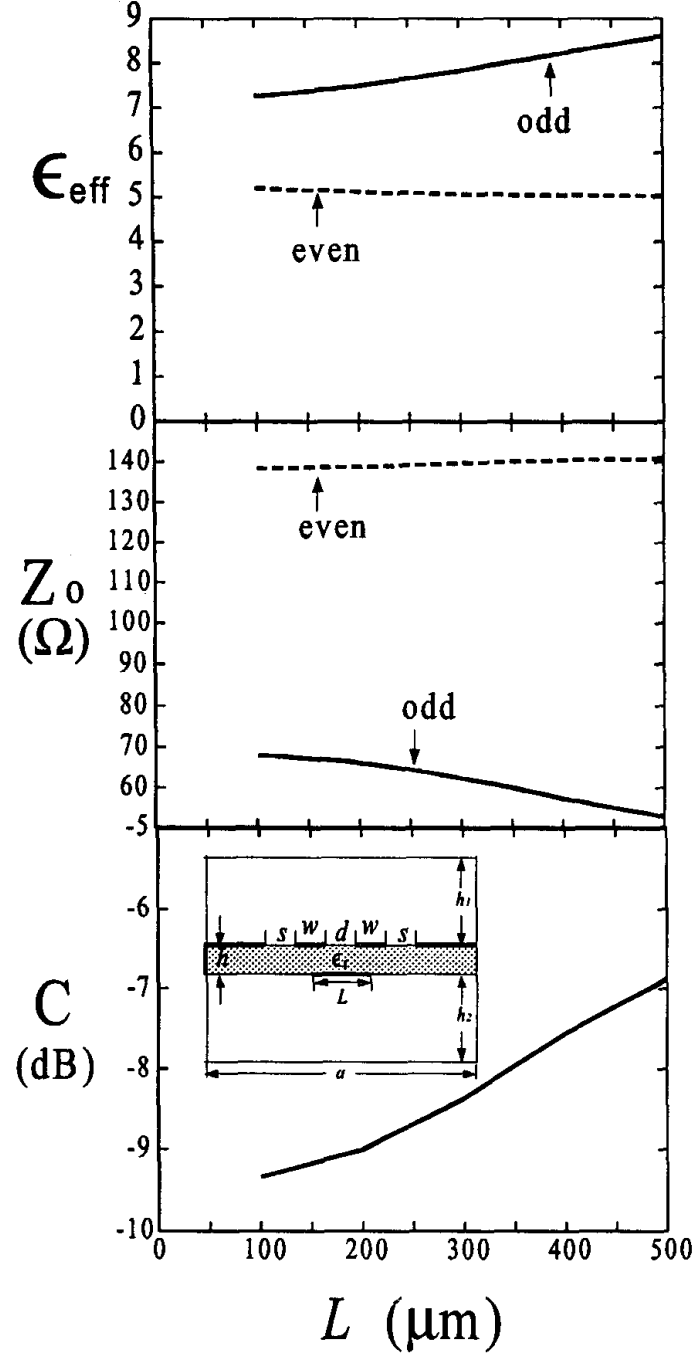

Fig. 3. Effect of back-conductor width $L$ on effective dielectric constants, characteristic impedances, and coupling coefficient of the conductor-backed edge-coupled CPW structure $(d=300 \mu m, f=3 G H z)$.

with reasonable line spacing $d$, a finiteextent backed conductor is added in this paper.

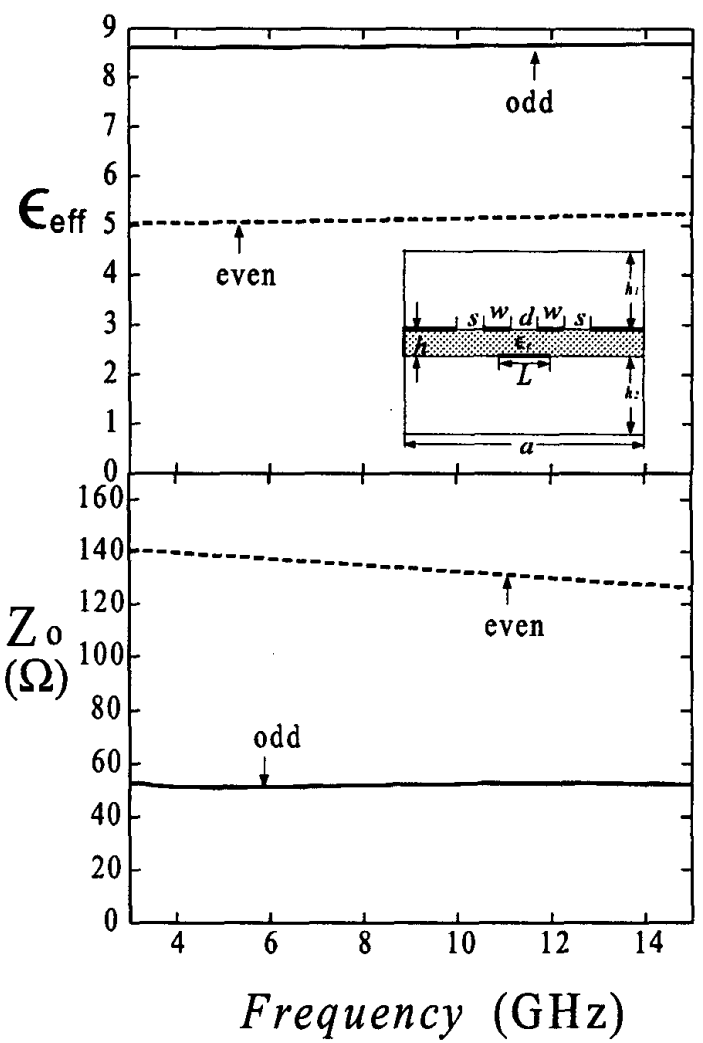

Fig. 4. Frequency dependence of effective dielectric constants and characteristic impedances of the conductor-backed edgecoupled CPW structure $(d=300 \mu m, L=$ $500 \mu \mathrm{m})$.

With the line spacing $d$ fixed as $300 \mu m$, Fig. 3 shows the effect of backed condurtor width $L$ on the conductor-backed edgecoupled (YPW structure (Fig. 1). The difference in $\epsilon_{\text {eff }}$ 's and $Z_{0}$ 's between two CPW modes becomes larger as $L$ is increased. The coupling coefficient $C$ is larger than $-7 \mathrm{~dB}$ as $L>500 \mu \mathrm{rr}$.

Fig. 4 shows the frequency dependenes of effective dielectric constants and charac- 


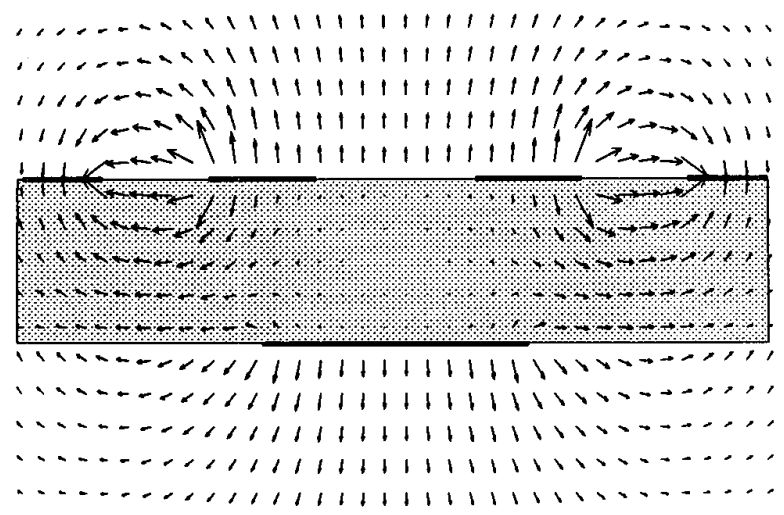

(a)

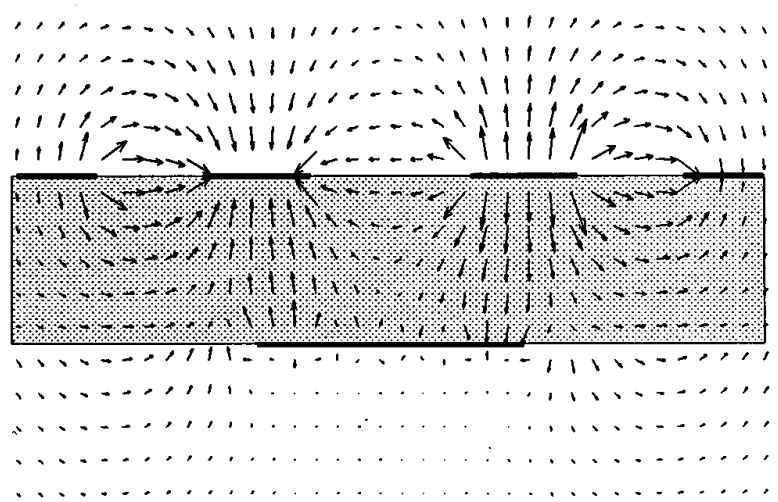

(b)

Fig. 5. Electric field distributions for (a) even CPW mode and (b) odd CPW mode $(L=500 \mu m, d=300 \mu m, f=3 G H z)$.

teristic impedances of the conductor-backed edge-coupled CPW structure. The dispersion effect is not serious for these two parameters as the backed conductor is added.

The electric field distributions for both even and odd CPW modes with $L=500 \mu \mathrm{m}$ are shown in Fig. 5. 'I'his figure shows that the backed conductor offers additional coupling mechanism to the proposed coupled structure.

\section{CONCLUSION}

In this work, a full-wave analysis is presented to characterize the effective dielectric constants, characteristic impedances, and coupling coefficient of the proposed conductor-backed edgecoupled CPW structure. To increase the coupling effect of the conventional edgecoupled CPW structure with reasonable line spacing, a finite-extent backed conductor is introduced in the proposed coupled CPW structure. The dispersion effect of the new structure is not serious and the coupling coefficient is found to be increased as the width of the backed conductor is increased.

\section{REFERENCES}

[1] C. P. Wen, "Coplanar-waveguide directional couplers," IEEE Trans. Microwave Theory Tech., vol. 18, pp. 318-322, June 1970.

[2] M. R. Lyons and C. A. Balanis, "I'ransient coupling reduction and design considerations in edge-coupled coplanar waveguide couplers," IEEE Trans. Microwave Theory Tech., vol. 44, pp. 778 783, May 1996.

[3] J. Y. Ke and C. H. Chen, "Dispersion and attenuation characteristics of coplanar waveguides with finite metallization thickness and conductivity :" IEEE Trans. Microwave Theory Tech., vol. 43, pp. 1128-1135, May 1995. 